\title{
Sites of M-CSF messenger RNA production in bone marrow trephine biopsy specimens and long term cultures demonstrated by non- isotopic in situ hybridisation
}

\author{
B S Wilkins, D B Jones
}

\begin{abstract}
Aim-To develop methods of messenger RNA (mRNA) in situ hybridisation (ISH) for use with routinely processed bone marrow trephine biopsy specimens, decalcified using formic acid, and long term cultures in order to demonstrate sites of synthesis of mRNA encoding monocyte colony stimulating factor (M-CSF). Methods-Biotinylated oligonucleotide probes, directed against target sequences within M-CSF mRNA, were hybridised with sections from bone marrow trephine biopsy specimens and detected using Streptavidin-biotin alkaline phosphatase complex formation. Validation of results included demonstration of total mRNA and unrelated mRNA species in adjacent sections, with appropriate negative controls. Minor technical modifications were required to perform ISH with long term bone marrow cultures.
\end{abstract}

Results-M-CSF mRNA was demonstrated successfully in trephine biopsy specimens and long term cultures. Biopsy specimens varied in their requirement for predigestion with proteinase $K$ and in the strength of the final reaction product, presumably due to variation in fixation. $M$ CSF mRNA was present in myelocytes and promonocytes. No stromal production of M-CSF mRNA was detected in biopsy specimens. ISH using long term bone marrow cultures confirmed production of $M$ CSF mRNA by developing monocytes and macrophages. Weak M-CSF mRNA expression was also seen in stromal fibroblasts.

Conclusions-ISH can be performed successfully with formic acid decalcified bone marrow trephine biopsy specimens and long term cultures. The presence of $M$ CSF mRNA in myelomonocytic cells suggests that an autocrine mechanism contributes to monocyte differentiation. The absence of detectable M-CSF mRNA in biopsy stroma and its presence in stromal fibroblasts within bone marrow cultures probably reflects reduced sensitivity of ISH following tissue fixation and processing.

(f Clin Pathol: Mol Pathol 1995;48:M35-M39)

Keywords: Bone marrow, in situ hybridisation, growth factors.
Developing haemopoietic cells in human bone marrow show preferential localisation within different areas of the intertrabecular spaces. ${ }^{1}$ The physiological basis of this spatial organisation is not known. Evidence from studies of cultured bone marrow indicate that growth factors needed for haemopoietic differentiation become bound to heparan sulphate proteoglycan in the extracellular matrix after secretion by stromal cells. ${ }^{23}$ Multipotential haemopoietic cells are able to interact with growth factors such as granulocyte-monocyte colony stimulating factor (GM-CSF) in this bound form and differentiate accordingly. Stromal localisation of growth factors within the extracellular matrix may create microenvironmental niches in which presentation of a particular combination of growth factors directs haemopoietic differentiation along specific pathways in a spatially restricted manner. ${ }^{4}$

Demonstration of the distribution of haemopoietic growth factors in stromal layers of long term bone marrow cultures or trephine biopsy specimens would provide powerful evidence for the existence of such microenvironmental niches. Immunohistochemical staining to investigate growth factor distribution within bone marrow has several limitations. Such an approach does not permit discrimination between sites of synthesis, stromal binding and uptake by responding cells. In long term culture systems diffusion of secreted growth factors may occur, which may not be relevant to the situation in vivo. Finally, currently available antibodies directed against haemopoietic growth factors have been developed primarily for enzyme linked immunosorbent assays or bioassays and most are poor reagents for immunohistochemistry.

In situ hybridisation has been used successfully in a variety of tissues to demonstrate sites of production of messenger RNA (mRNA) encoding proteins. Messenger RNA can be stained in routinely fixed and processed tissues as well as fresh cells and frozen tissue. Trephine biopsy specimens of bone marrow differ from many histological samples in that they require decalcification before being processed for embedding in wax. This is usually achieved by exposure to acid or to a chelating agent such as EDTA. Few studies have addressed the question of mRNA preservation in decalcified tissue samples and only one published report exists which describes mRNA in situ hybridisation in bone marrow trephine biopsy specimens. ${ }^{5}$ In 
that study mRNAs for $\kappa$ and $\lambda$ light chains were demonstrated successfully by in situ hybridisation using biotin labelled oligonucleotide probes with EDTA decalcified biopsies. In Southampton trephine biopsy specimens are decalcified using a $5 \%$ solution of formic acid in $10 \%$ formalin. We have used biotin labelled 30 mer oligonucleotide probes directed against mRNA encoding monocyte/macrophage colony stimulating factor (M-CSF) to demonstrate the feasibility of performing in situ mRNA hybridisation in tissue decalcified using formic acid and to investigate the sites of production of M-CSF within bone marrow. We have also adapted the method successfully for application to intact adherent cell layers of long term bone marrow cultures.

\section{Methods}

Bone marrow trephine biopsy specimens showing no morphological abnormalities were se-
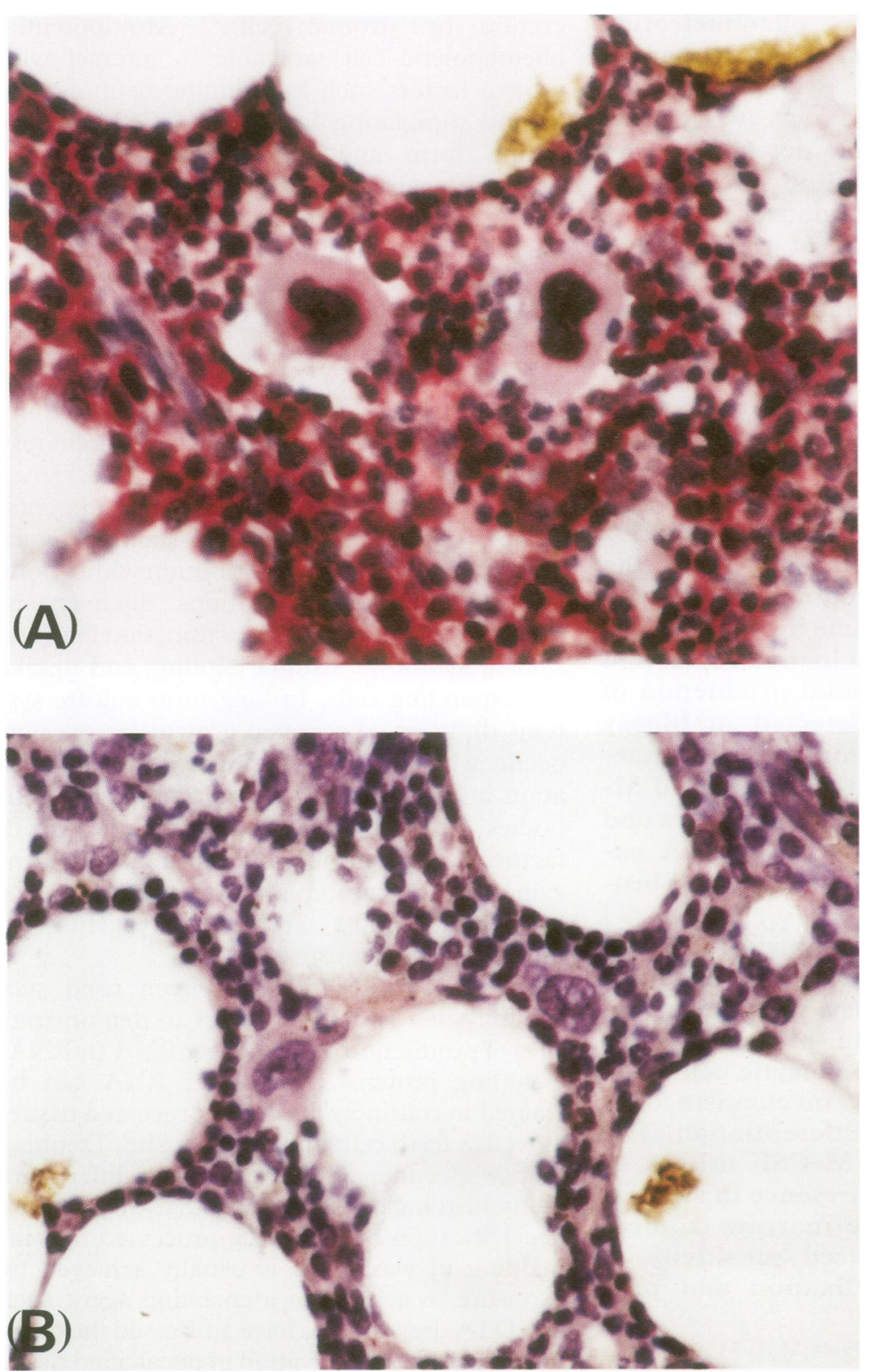

Figure 1 (A) Demonstration of total cellular $m R N A$ in a bone marrow trephine biopsy using a 25 mer oligo dT probe. (B) Control section from the same biopsy showing lack of non-specific signal when the 25 mer oligo $d A$ probe was used.

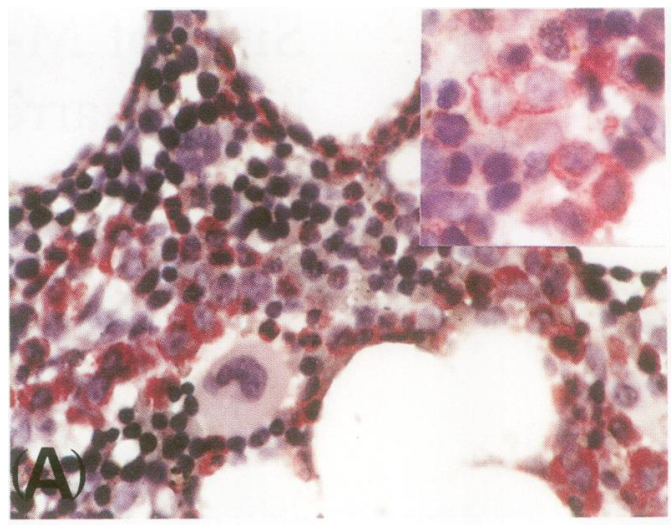

lected from the diagnostic files of the Pathology Department of the Southampton University Hospitals Trust. These biopsies had been performed for investigation of possible marrow involvement by metastatic carcinoma or nonHodgkin's lymphoma but had been found to show no pathology. Biopsy specimens from five cases each of various myelodysplastic syndromes (refractory anaemia, refractory anaemia with ringed sideroblasts, refractory anaemia with excess blasts, and chronic myelomonocytic leukaemia), five cases of acute myeloid leukaemia of French-American-British (FAB) classification AML-M2, and five of AML-M5 were also studied.

All biopsy specimens had been fixed in $10 \%$ neutral buffered formalin, decalcified in 5\% formic acid in formalin and processed by routine methods into paraffin wax blocks. Sections $5 \mu \mathrm{m}$ thick were cut and mounted onto slides coated with a $1 \%$ solution of 3-aminopropyltriethoxysilane.

Aspirated bone marrow from patients undergoing staging investigations for non-Hodgkin's lymphoma was collected into sterile Iscove's medium (Gibco BRL, UK) containing $25 \mathrm{U} / \mathrm{ml}$ heparin. In all cases studied subsequent morphological analysis of the patients' aspirate and trephine samples showed no evidence of lymphoma or other pathology. Each aspirate sample was layered onto Lymphoprep (Nycomed, Oslo, Norway) and centrifuged for 30 minutes at $2000 \mathrm{rpm}$. Nucleated cells were 


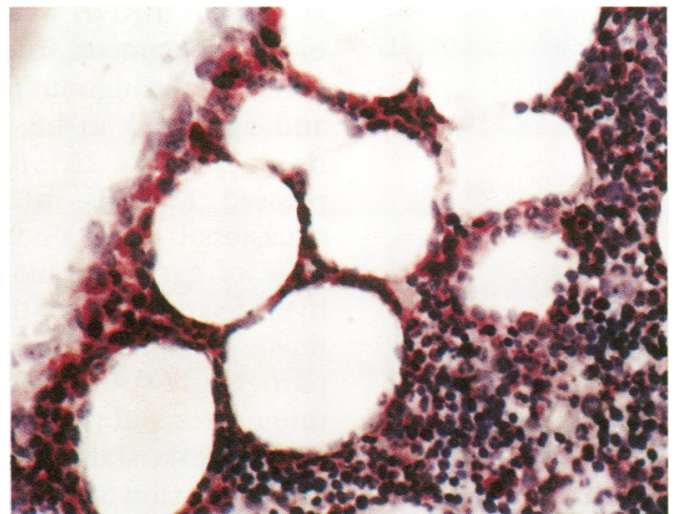

Figure 3 Pattern of hybridisation when IL-4 antisense oligonucleotide probe was used. Strong expression is seen within the paratrabecular zone of immature granulocytes, with weaker staining of later granulocytes.

then collected from the medium-Lymphoprep interface and washed once in Iscove's medium by centrifugation for 10 minutes at $1100 \mathrm{rpm}$. The cell pellet was then resuspended and Dexter-type cultures ${ }^{6}$ were established using $1 \cdot 0-1 \cdot 25 \times 10^{6}$ cells $/ \mathrm{ml}$ in Iscove's medium containing benzyl penicillin $(600 \mu \mathrm{g} / \mathrm{ml})$ and Streptomycin sulphate $(200 \mu \mathrm{g} / \mathrm{ml})$, supplemented with $10 \%$ fetal calf serum, $10 \%$ horse serum, and $10^{-7} \mathrm{M}$ hydrocortisone (as sodium hemisuccinate salt). Cultures were grown directly onto glass slides in the form of slide-flasks (Nunc Inc., Illinois, USA). They were grown at $33^{\circ} \mathrm{C}$ in $5 \% \mathrm{CO}_{2}$ in air for six to 12 weeks, with half of the medium being replaced at weekly intervals. Culture was terminated by gently washing once with phosphate buffered saline (PBS), $\mathrm{pH} 7 \cdot 6$, followed by air-drying.

In situ hybridisation was performed with a synthetic 30 base oligonucleotide probe antisense to a selected sequence of the published complementary DNA (cDNA) sequence for M-CSF. This probe had a GC content of $72 \%$ and a theoretical melting point of $82^{\circ} \mathrm{C}$.

Biotinylated dUTP was used to label the $3^{\prime}$

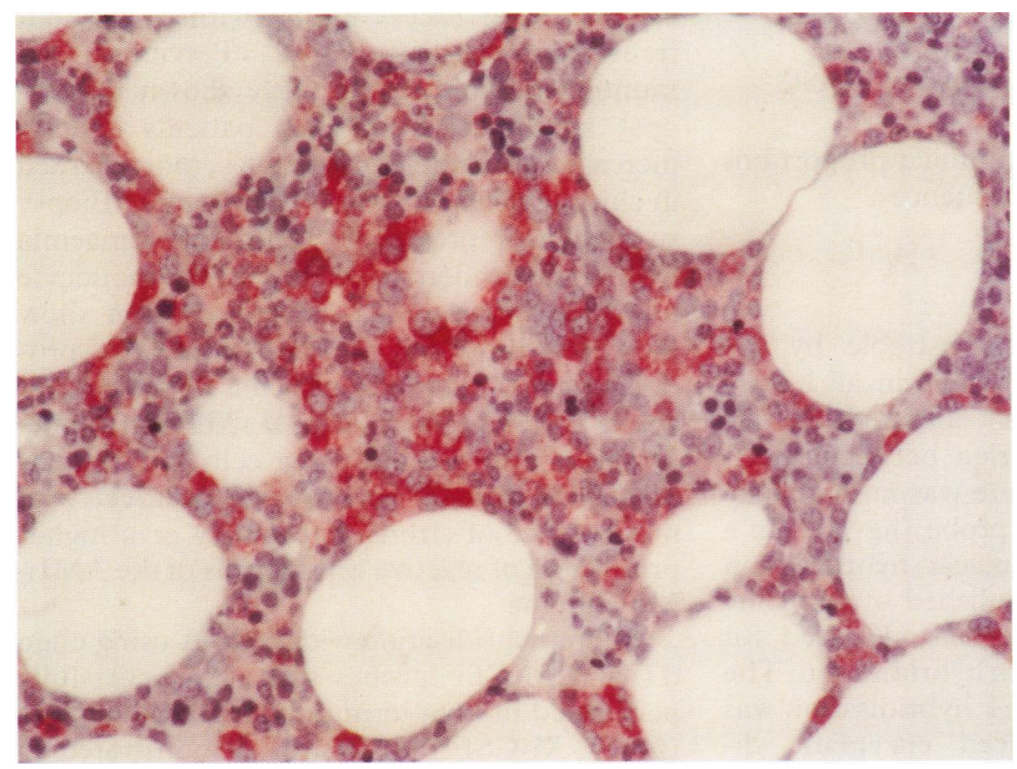

Figure 4 Low power photomicrograph of bone marrow from a case of chronic myelomonocytic leukaemia hybridised with M-CSF antisense oligonucleotide probe. Large numbers of immature granulocytes and monocytes are present expressing M-CSF mRNA. terminal of the oligonucleotide, catalysed by terminal deoxynucleotidyl transferase. Hybridisation was performed according to the method of Pringle et al. ${ }^{5}$ Briefly, trephine biopsy sections were dewaxed in xylene, rehydrated through graded alcohols, permeabilised by immersion in $0 \cdot 2 \mathrm{~N}$ hydrochloric acid followed by PBS containing Triton X-100, digested using proteinase $\mathrm{K}$, and post-fixed in $0.4 \%$ paraformaldehyde in PBS at $4^{\circ} \mathrm{C}$ for 20 minutes. Air-dried bone marrow cultures were divided into two to four sections using a hydrophobic pen (Dako) but required no further pretreatment before fixation in $4 \%$ paraformaldehyde in PBS at $4^{\circ} \mathrm{C}$ for 10 minutes.

Biopsy sections and cultures were prehybridised for 30 minutes at $37^{\circ} \mathrm{C}$ in hybridisation buffer consisting of $50 \%$ formamide, $5 \%$ polyethylene glycol, $12 \% 5 \mathrm{M}$ $\mathrm{NaCl}, 10 \% 10 \times \mathrm{PE}$ buffer $(0.5 \mathrm{M}$ Tris $-\mathrm{HCl}$, $1 \%$ sodium pyrophosphate, $2 \%$ polyvinylpyrolidone, $2 \%$ Ficoll 400000 , and $50 \mathrm{mM}$ EDTA), and $2 \%$ denatured salmon sperm DNA in ultrapure water. This solution was then replaced by similar buffer containing probe at a concentration of $2 \mathrm{ng} / 50 \mu \mathrm{l}$. Clean coverslips were applied to minimise evaporation and hybridisation was performed overnight at $37^{\circ} \mathrm{C}$ in a humid chamber. Slides were then rinsed with $2 \times$ standard saline citrate (SSC) and stringency washes were performed with $0.2 \times \mathrm{SSC}$ at $42^{\circ} \mathrm{C}$ and room temperature. Bound probe was detected using Streptavidinbiotin alkaline phosphatase complexes (Dako) with Fast Red/naphthol ASB1-phosphate as substrate for the final coloured product. Sections and cultures were then mounted using CrystalMount (Biogenesis, Bournemouth, $\mathrm{UK})$, dried at $80^{\circ} \mathrm{C}$ and then double-mounted with DPX $(\mathrm{BDH})$ for permanent application of coverslips.

All buffers and solutions used throughout the hybridisation procedures were prepared with ultrapure water, by reverse osmosis, and treated with $0.1 \%$ diethyl pyrocarbonate to destroy RNase enzymes. With the exception of the veronal acetate buffer used for the final colour reaction, all buffers were sterilised by autoclave. All glassware was washed at $80^{\circ} \mathrm{C}$ and rinsed with ultrapure water. Gloves were worn at all times while preparing reagents and performing hybridisation reactions.

In all cases hybridisation was performed with a sense probe complementary to the antisense M-CSF oligonucleotide as a negative control. Each case was hybridised additionally with a 25 mer of dT to demonstrate the poly-A tails of all intact $\mathrm{mRNA}$ species present and with a negative control 25 mer of dA. Hybridisation with buffer containing no probe was also performed in all cases. A subset of morphologically normal biopsy specimens was hybridised with 30 mer sense and antisense probes directed against interleukin-1 (IL-1), IL-4, IL-5, interferon- $\gamma$ (IFN $\gamma$ ), and tumour necrosis factor $\alpha$ (TNF $\alpha$ ) to demonstrate mRNA species unrelated to M-CSF. These probes had similar GC contents to that of the M-CSF probe used. The IL-1, IL-4 and IL-5 probes consisted of single sequences, while the IFN $\gamma$ and TNF $\alpha$ 

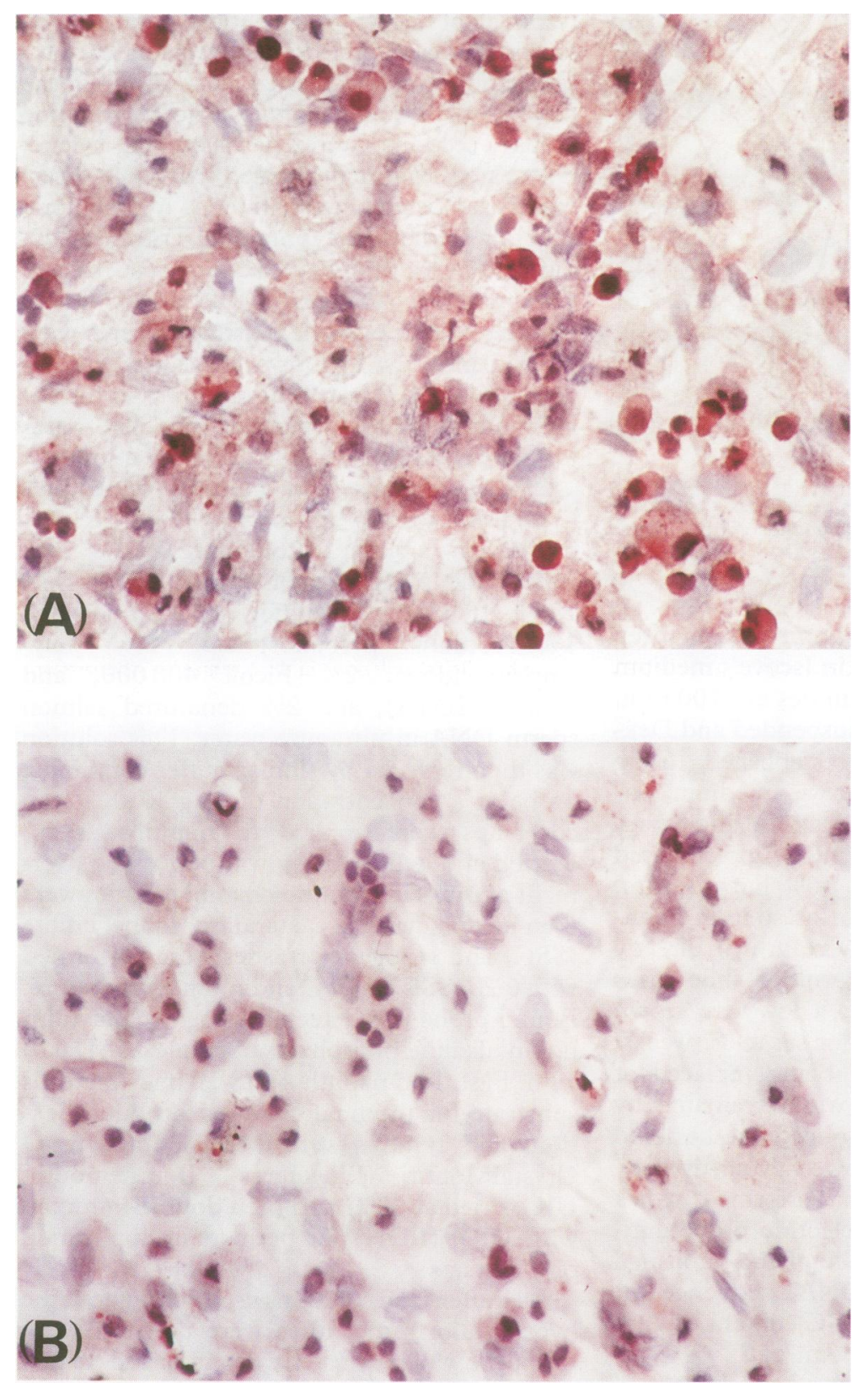

Figure 5 (A) Six week old bone marrow culture hybridised with a M-CSF antisense oligonucleotide probe. There is a weak positive staining of stromal fibroblasts but much stronger staining of monocytes and small macrophages adherent to the fibroblastic layer. (B) Control area of the same bone marrow culture hybridised with a M-CSF sense oligonucleotide probe. A very low level of non-specific background staining is present.

probes were cocktails of equimolar proportions of three different 30 mer sequences.

\section{Results}

Messenger RNA was demonstrable by hybridisation with oligo dT probe in all biopsy specimens studied (fig 1A), although the intensity of the reaction varied between specimens. In most samples there was no reactivity with the control oligo $\mathrm{dA}$ probe (fig 1B). In a few, however, spurious nuclear hybridisation was detected. This was abolished by reducing the concentration of proteinase $\mathrm{K}$ used for digestion of sections prior to hybridisation. The specific signal from oligo dT hybridisation was maintained despite reduced enzymatic digestion, suggesting that some specimens, possibly because of variations in fixation, are highly sensitive to the enzyme.
M-CSF mRNA was also demonstrable in all biopsy specimens studied. Positive cells were scattered throughout the intertrabecular spaces and appeared to be promonocytes and promyelocytes (fig 2A). Not all promyelocytes expressed M-CSF mRNA, however, as only occasional cells amongst the paratrabecular zone of early granulocytes were positive. No hybridisation with stromal cells was seen in most specimens. In occasional cases with a particularly strong hybridisation signal in promonocytes and promyelocytes, there was equivocal, weak, staining of spindle cells. Stromal cell expression of M-CSF mRNA may be below the threshold of our detection system in most specimens.

In most biopsy specimens no reactivity was seen with the M-CSF sense probe (fig 2B). Eosinophils and their precursors were problematical in a few cases, giving non-specific reactions with sense and antisense M-CSF probes. This could be overcome by using dextran in place of polyethylene glycol in the hybridisation buffer, but only at the expense of a reduction in strength of the specific signal. As eosinophil precursors are readily recognised morphologically in trephine biopsy specimens, it was not difficult to exclude them from analysis in the few samples where non-specific reactivity occurred. Of the other cytokine probes tested, TNF $\alpha$ and IL-4 showed reactivity with haemopoietic cells. Tumour necrosis factor mRNA was present in a minor population of large, seemingly primitive cells dispersed throughout the marrow spaces. Interleukin-4 mRNA was present in most promyelocytes and myelocytes comprising the paratrabecular zones of early granulopoiesis (fig 3). For both TNF $\alpha$ and IL-4, control sense oligonucleotide sequences gave no reaction.

Compared with morphologically normal biopsy specimens, increased numbers of $\mathrm{M}$-CSF mRNA producing cells were present in most specimens from patients with myelodysplastic syndromes, particularly in cases of chronic myelomonocytic leukaemia and refractory anaemia with excess blasts (with or without transformation) (fig 4). Previous immunophenotypic studies have shown that biopsy specimens from such patients contain increased numbers of monocytes, most marked in chronic myelomonocytic leukaemia. ${ }^{8}$ Biopsy specimens representing refractory anaemia with ringed sideroblasts, in which monocyte numbers are generally normal, did not show increased numbers of M-CSF mRNA producing cells. Examples of acute myeloid leukaemia of FAB subtypes AML-M2 and AMLM5 were also studied. Blast cells in both categories of disease were weakly positive, with no evidence of stronger reactivity or a higher proportion of reactive cells in cells of the AMLM5 subtype.

In situ hybridisation with mRNA using oligo dT and M-CSF probes was also successfully performed in long term bone marrow cultures (fig 5). M-CSF mRNA was demonstrated in developing monocytes and in small round macrophages adherent to the stromal layer, but not in elongated, highly spread macrophages 
known from immunophenotypic studies to be present in the stroma (Wilkins BS, unpublished observations). A weak M-CSF mRNA signal was also detected in spindle shaped and polygonal fibroblastic cells which predominate in bone marrow culture stroma. No reactivity was seen with the small, primitive cells in haemopoietic colonies.

\section{Discussion}

This study has shown that mRNA is preserved in bone marrow trephine biopsy specimens despite fixation and decalcification. Previously, mRNA in situ hybridisation has only been reported with trephine specimens decalcified using calcium chelating agents. ${ }^{5}$ That report related to $\kappa$ and $\lambda$ immunoglobulin light chain mRNA in plasma cells of multiple myeloma, expressed in high copy number within the neoplastic cells. Our study has shown that mRNA for M-CSF, a cytokine growth factor likely to be expressed in low copy number per cell, ${ }^{9}$ can also be detected using a non-isotopic in situ hybridisation technique.

$\mathrm{M}-\mathrm{CSF}$ is required for the differentiation of monocytes within bone marrow and for various aspects of macrophage maturation and activation. ${ }^{10}$ The presence of M-CSF in cells of promonocyte/promyelocyte morphology in the trephine biopsy specimens, and in monocytes and macrophages in the bone marrow cultures suggests that an autocrine loop of M-CSF production and response may operate during monocyte differentiation. The apparent lack of precise correlation between cell types expressing M-CSF mRNA in biopsy specimens and cultures is partly caused by the difficulty of categorising relatively undifferentiated cells in haemopoietic colonies in bone marrow cultures. The lack of M-CSF signal in stromal fibroblasts in most of our trephine biopsy specimens was probably because of reduced sensitivity of the technique and/or reduced mRNA preservation in fixed, decalcified tissue compared with the bone marrow cultures.

It has been proposed that stromal microenvironments exist for haemopoiesis in culture, ${ }^{4}$ permitting or directing differentiation along restricted pathways. No preferential localisation of M-CSF mRNA production was seen in areas of stroma showing colony formation compared with areas not forming colonies. This is in keeping with the results of previous immunohistochemical studies of monocytopoiesis in bone marrow trephine biopsy specimens which showed that monocyte production, unlike granulocyte production, occurs in a dispersed fashion throughout the intertrabecular spaces. ${ }^{8}$ We are currently studying the distribution of GM-CSF and G-CSF mRNA within cells in bone marrow trephine biopsy specimens and long term cultures to discover whether or not production of these growth factors is spatially restricted.

The authors wish to thank the Cancer Research Campaign, who funded this study. We are also grateful to Dr D Choudhury and other clinical colleagues in the Haematology Department, Southampton University Hospitals Trust, who provided bone marrow aspirate samples for culture.

1 Wilkins BS. Histology of normal haemopoiesis: bone marrow histology I. $\mathcal{f}$ Clin Pathol 1992;45:645-9.

2 Long MW. Blood cell cytoadhesion molecules. Exp Hematol 1992;20:288-301.

3 Gordon MY, Riley GP, Watt SM, Greaves MF. Compartmentalisation of a haematopoietic growth factor (GMCSF) by glycosaminoglycans in the bone marrow miCSF) by glycosaminoglycans in the bone
croenvironment. Nature 1987;326:403-5.

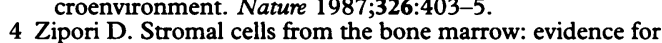
restrictive role in regulation of hemopoiesis. Eur $\mathcal{F}$ Haematol $1989 ; 42: 225-32$

5 Akhtar N, Ruprai A, Pringle JH, Lauder I, Durrant STS. In situ hybridisation detection of light chain mRNA in routine marrow trephines from patients with suspected myeloma. Br f Haematol 1989;73:296-301.

6 Coutinho LH, Gilleece MH, de Wynter EA, Will A, Testa NG. Clonal and long-term cultures using human bone marrow. In: Testa NG, Molineux G, eds. Haemopoiesis: practical approach. Oxford: Oxford University Press, 1993: practical

7 Pringle JH, Ruprai A, Kyte J, Potter L, Close PM, Lauder Pringle JH, Ruprai A, Kyte J, Potter L, Close PM, Lauder
I. In situ hybridisation demonstration of polyadenylated RNA sequences in formalin fixed paraffin sections using a biotinylated oligonucleotide poly (dT) probe. $\mathcal{F}$ Pathol 1989;158:279-86.

8 Wilkins BS, Jones DB. Cell-stroma interactions in monocytopoiesis. FEMS Microbiol Immunol 1992;105:347-54

9 Yamato K, El-Hajioui Z, Koeffler HP. Expression of hematopoietic growth factor RNAs in human mesenchymal cells from various organs. Leukemia Res 1991;15:551-8.

10 Stanley ER, Guilbert LJ, Tushinski RJ, Bartelmez SH. CSF-1 -A mononuclear phagocyte lineage-specific hemopoietic growth factor. $\mathcal{f}$ Cell Biochem 1983;21:151-9. 